\title{
Effect of prone positioning during mechanical ventilation on mortality among patients with acute respiratory distress syndrome: a systematic review and meta-analysis
}

\author{
Sachin Sud MD MSc, Jan O. Friedrich MD DPhil, Neill K. J. Adhikari MDCM MSc, Paolo Taccone MD, \\ Jordi Mancebo MD, Federico Polli MD, Roberto Latini MD, Antonio Pesenti MD, Martha A.Q. Curley RN PhD, \\ Rafael Fernandez MD, Ming-Cheng Chan MD, Pascal Beuret MD, Gregor Voggenreiter MD, \\ Maneesh Sud MD, Gianni Tognoni MD, Luciano Gattinoni MD, Claude Guérin MD PhD
}

\begin{abstract}
Background: Mechanical ventilation in the prone position is used to improve oxygenation and to mitigate the harmful effects of mechanical ventilation in patients with acute respiratory distress syndrome (ARDS). We sought to determine the effect of prone positioning on mortality among patients with ARDS receiving protective lung ventilation.
\end{abstract}

Methods: We searched electronic databases and conference proceedings to identify relevant randomized controlled trials (RCTs) published through August 2013. We included RCTs that compared prone and supine positioning during mechanical ventilation in patients with ARDS. We assessed risk of bias and obtained data on all-cause mortality (determined at hospital discharge or, if unavailable, after longest follow-up period). We used random-effects models for the pooled analyses.
Results: We identified 11 RCTs $(n=2341)$ that met our inclusion criteria. In the 6 trials $(n=$ 1016) that used a protective ventilation strategy with reduced tidal volumes, prone positioning significantly reduced mortality (risk ratio $0.74,95 \%$ confidence interval 0.59 $0.95 ; l^{2}=29 \%$ ) compared with supine positioning. The mortality benefit remained in several sensitivity analyses. The overall quality of evidence was high. The risk of bias was low in all of the trials except one, which was small. Statistical heterogeneity was low $\left(I^{2}<50 \%\right)$ for most of the clinical and physiologic outcomes.

Interpretation: Our analysis of high-quality evidence showed that use of the prone position during mechanical ventilation improved survival among patients with ARDS who received protective lung ventilation.

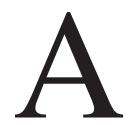
cute respiratory distress syndrome (ARDS) is a fulminant disease characterized by impaired oxygenation, pulmonary congestion and decreased lung compliance following a direct pulmonary insult, such as aspiration or pneumonia, or a systemic injury, such as sepsis or trauma. In many patients with ARDS, mechanical ventilation can further injure damaged lungs, ${ }^{1-3}$ leading to longterm morbidity and mortality. Although minimizing tidal volumes ${ }^{4}$ and optimizing positive end-expiratory pressure (PEEP) ${ }^{5}$ may reduce lung injury, mortality associated with ARDS remains high. ${ }^{6,7}$

Placing patients in the prone position for a portion of time each day during mechanical ventilation, first suggested in $1974,{ }^{8}$ is sometimes used as a protective lung strategy in patients with ARDS. ${ }^{9,10}$ Alveolar distension varies regionally because of gravity and anatomic relationships with the chest wall and heart. In the prone posi- tion, the volume of lung collapsed under its own weight and that of the heart is decreased relative to the supine position. ${ }^{10,11}$ Because pulmonary perfusion is preserved in both the ventral and dorsal lung regions, ventilation-perfusion matching is improved in the prone position. ${ }^{12}$ More homogenous dispersion of tidal volume in the prone position may minimize alveolar stretch and strain. ${ }^{10}$ Improvements in oxygenation may reduce the risk of death from hypoxia. . $^{13,14}$

Early randomized controlled trials (RCTs) of prone positioning did not show reductions in mortality. ${ }^{15,16}$ However, these trials included patients with mild ARDS, the duration of prone positioning each day was short, and protective lung ventilation (low tidal volumes) was not used. A subsequent meta-analysis suggested that prone positioning reduces mortality among patients with severe hypoxemia. ${ }^{13}$ Because of the availability of new data, ${ }^{17}$ we undertook a systematic review and meta-analysis, in collabora-
Competing interests: Jordi Mancebo received grants from Covidien and General Electric for research on proportional assist ventilation and functional residual capacity; he served on the data monitoring and safety board for a trial sponsored by Air Liquide, and on the steering committees for Faron Pharmaceuticals and ALung Technologies. Luciano Gattinoni was a member of an advisory board for KCI Medical. No other competing interests were declared.

This article has been peer reviewed.

Correspondence to: Sachin Sud, sachin.sud @ trilliumhealthpartners.ca

CMAJ 2014. DOI:10.1503 /cmaj.140081 
tion with all of the primary investigators, to determine whether a strategy incorporating the prone position for a portion of each day, compared with supine position alone, decreases mortality among patients with ARDS receiving protective lung ventilation.

\section{Methods}

\section{Literature search}

We conducted a systematic review using a previously described protocol with prespecified selection criteria, outcome measures and analysis plan. ${ }^{13}$ Amendments to the protocol for the current review are described in Appendix 1 (available at www.cmaj.ca/lookup/suppl/doi:10.1503 /cmaj.140081/-/DC1). We systematically searched multiple sources to identify RCTs that compared prone positioning with supine positioning during mechanical ventilation in patients with ARDS. To identify relevant trials published since the earlier systematic review, ${ }^{13}$ we searched the electronic databases MEDLINE, Embase and CENTRAL (the Cochrane Central Register of Controlled Trials) for articles published from June 2009 to August 2013. We also searched the bibliographies of included studies and review articles, as well as the conference proceedings of the American Thoracic Society (1994-2013), the Society of Critical Care Medicine (1994-2013) and the European Society of Intensive Care Medicine (1994-2013). Finally, we searched the ClinicalTrials.gov registry and the Current Controlled Trials database for unpublished and ongoing trials. No language restrictions were applied. Details of our search strategy are described in Appendix 2 (available at www.cmaj.ca/lookup /suppl/doi:10.1503/cmaj.140081/-/DC1).

\section{Study selection}

Three of us (S.S., J.O.F. and M.S.), who were not blinded to the study authors or results, independently evaluated the studies for inclusion and resolved differences through consensus.

We included RCTs and quasi-randomized trials (e.g., trials allocating patients in alternating fashion or by hospital registry number) if they met the following criteria: they enrolled adults or postneonatal children with ARDS supported by mechanical ventilation; they used prone positioning in the experimental group and supine positioning alone in the control group; and they reported any of the primary or secondary outcomes described in the next section. We included trials that used either the older ${ }^{18}$ or more recent ${ }^{19}$ definition of ARDS. For trials that enrolled patients with other forms of hypoxemic respiratory failure, we asked the primary investigators to supply mortality data for patients who had ARDS at the time of enrolment.

We excluded crossover trials. We also excluded physiologic studies lasting 48 hours or less, reasoning that a brief intervention period would not affect clinical outcomes.

\section{Data extraction and quality assessment}

The 3 of us involved in selecting the studies also independently abstracted data on study methods, details of prone positioning and mechanical ventilation, and study outcomes. Disagreements were resolved through consensus.

The primary outcome was all-cause mortality. For each study, mortality was determined at hospital discharge or, if not available, the longest duration of follow-up. For the primary analysis, we included only trials in which all patients received protective lung ventilation (defined as tidal volume $<8 \mathrm{~mL} / \mathrm{kg}$ of predicted body weight), ${ }^{4}$ because we felt studies that did not use protective ventilation might bias results to the null $1^{20,21}$ and would be less relevant to current clinical practice. Studies that did not mandate protective lung ventilation were included in the analyses of secondary outcomes.

Secondary outcomes included change in oxygenation and adverse events (ventilator-associated pneumonia, pressure ulcers, obstruction of endotracheal tube, unplanned extubation, unplanned removal of central venous catheters or arterial lines, unplanned removal of chest tubes, pneumothorax and cardiac arrest).

For the assessment of methodologic quality and risk of bias, we abstracted the following data: randomization methods and allocation concealment; number of withdrawals and losses to follow-up after randomization; crossovers between assigned groups; blinding of outcome assessors; and whether the trial was stopped early because of evidence of benefit. We assessed risk of bias using a modified version of the Cochrane risk-of-bias instrument. ${ }^{22}$ Because blinding of caregivers, patients and family members was not possible in the trials, we determined whether important cointerventions (e.g., weaning, sedation and paralysis) were standardized or applied equally in the treatment and control groups. We assessed the quality of evidence for our primary outcome using the GRADE approach (Grading of Recommendations Assessment, Development and Evaluation). ${ }^{23}$

Primary investigators collaborated in this systematic review by confirming original trial data, providing previously unpublished data for subgroups of patients, and clarifying data and methods.

\section{Data synthesis}

We performed 2 subgroup analyses. First, we hypothesized that maximizing the daily duration 
of prone positioning would optimize lung protection. We compared trials in which prone positioning was prolonged $(\geq 16 \mathrm{~h} / \mathrm{d})$ with those in which the duration was shorter. Second, we hypothesized that prone positioning would be of greater benefit in patients with more severe hypoxemia at baseline. We compared the effect of prone positioning on mortality among patients who had severe hypoxemia (baseline ratio of partial pressure of arterial oxygen to fraction of inspired oxygen $\left[\mathrm{PaO}_{2} / \mathrm{FIO}_{2}\right]<100 \mathrm{~mm} \mathrm{Hg}$ ) with mortality among patients who had moderate hypoxemia $\left(\mathrm{PaO}_{2} / \mathrm{FIO}_{2}\right.$ ratio $100-199 \mathrm{~mm} \mathrm{Hg}$ ) and those who had mild hypoxemia $\left(\mathrm{PaO}_{2} / \mathrm{FIO}_{2}\right.$ ratio $\left.200-299 \mathrm{~mm} \mathrm{Hg}\right) .{ }^{19}$

Finally, we analyzed the effect of prone positioning on oxygenation by obtaining data on the mean $\mathrm{PaO}_{2} / \mathrm{FIO}_{2}$ ratios on the first, second and third day after randomization for each treatment group.

We analyzed all outcomes using the intentionto-treat approach. We aggregated outcomes data at the trial level and performed statistical calculations using Review Manager software (RevMan version 5.1; Nordic Cochrane Centre, Cochrane Collaboration, 2011) and Stata software (Stata Statistical Software, release 9.2; StataCorp, 2006). For the pooled analyses, we used random-effects models, which incorporate between-study variation and generally result in wider confidence intervals than fixed-effects models do when heterogeneity is present. We reported continuous outcomes as ratios of means ${ }^{24}$ (a measure of relative change) and binary outcomes as risk ratios (RRs). For each subgroup analysis, we tested for interaction between the RR for mortality in each subgroup, which tests the null hypothesis that the treatment effect in each subgroup is the same. All statistical tests were 2 -sided. We considered a $p$ value of less than 0.05 to be statistically significant and reported individual trial and summary results with $95 \%$ confidence intervals (CIs).

We assessed between-study heterogeneity for each outcome using the $I^{2}$ statistic. ${ }^{25}$ We considered statistical heterogeneity to be low if the $I^{2}$ value was $25 \%-49 \%$, moderate if $50 \%-74 \%$ and high if $75 \%$ or greater.

We assessed publication bias using the Begg rank correlation test $\mathrm{t}^{26}$ and the Peters regression test. ${ }^{27}$ Given the low power of these tests, we considered a $p$ value less than 0.1 to indicate publication bias.

\section{Results}

\section{Search results and study characteristics}

In addition to the 15 RCTs from the previous meta-analysis, ${ }^{13}$ we identified 238 potentially eligible reports through searching electronic bibliographic databases. After screening the titles and abstracts and removing duplicate records, we reviewed 22 articles in full. We excluded 11 trials, 6 of which had intervention periods that lasted 48 hours or less, ${ }^{28-33}$ which left 11 trials $^{14-17,34-40}$ for the meta-analysis (Figure 1). Reviewers were in total agreement about the included studies.

The characteristics of the 11 included trials are summarized in Table 1 and in Appendix 3 (available at www.cmaj.ca/lookup/suppl/doi:10.1503 /cmaj.140081/-/DC1). A total of 2341 patients were enrolled (median 102, range 16-802); children were included in 1 trial $(n=102)$. Overall, the median $\mathrm{PaO}_{2} / \mathrm{FIO}_{2}$ ratio at enrolment was 118 (range 100-326) $\mathrm{mm} \mathrm{Hg}$, the median $\mathrm{FIO}_{2}$ was $73 \%$ (range $49 \%-88 \%$ ), and the median PEEP was 10 (range 7-13) $\mathrm{cm} \mathrm{H}_{2} \mathrm{O}$. The duration of ARDS before enrolment was less than 72 hours in 8 trials, ; $^{14,17,34-39} 2$ studies did not limit the duration before enrolment. ${ }^{15,16}$ One trial included 802 patients with acute hypoxemic respiratory failure, of whom 413 had ARDS. Another included 53 patients with a Glasgow Coma Score of 9 or less, of whom 7 had ARDS. All of the RCTs, except one small study, defined ARDS according to the American-European Consensus Conference definition, with varying thresholds for the $\mathrm{PaO}_{2} / \mathrm{FIO}_{2}$ ratio.

Prone positioning was used a median of 17 hours per day (range 4-24 h) for 4.6 days (range 4-10 d) and continued until prespecified criteria for clinical improvement were met $\left(9\right.$ trials $\left.^{14,16,17,34-39}\right)$ or

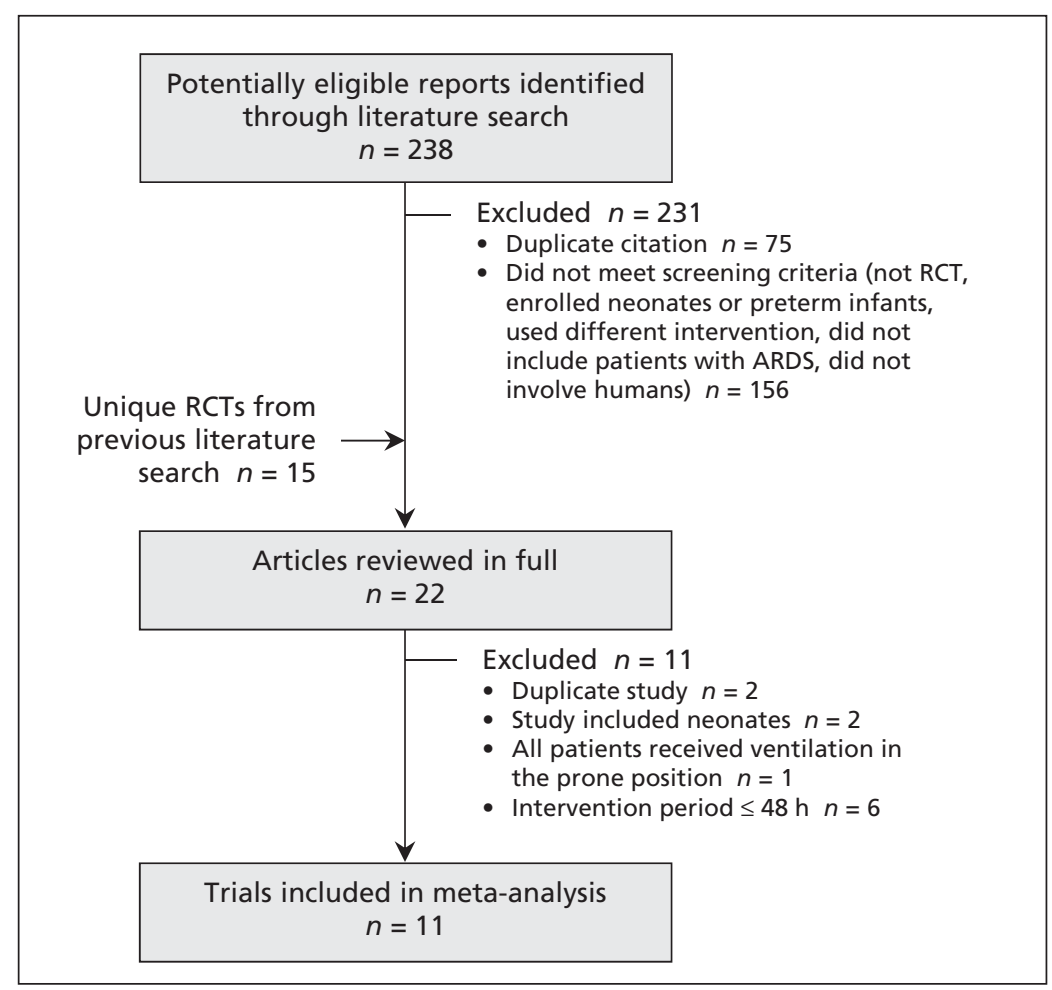

Figure 1: Selection of randomized controlled trials (RCTs) for the meta-analysis. ARDS = acute respiratory distress syndrome. 


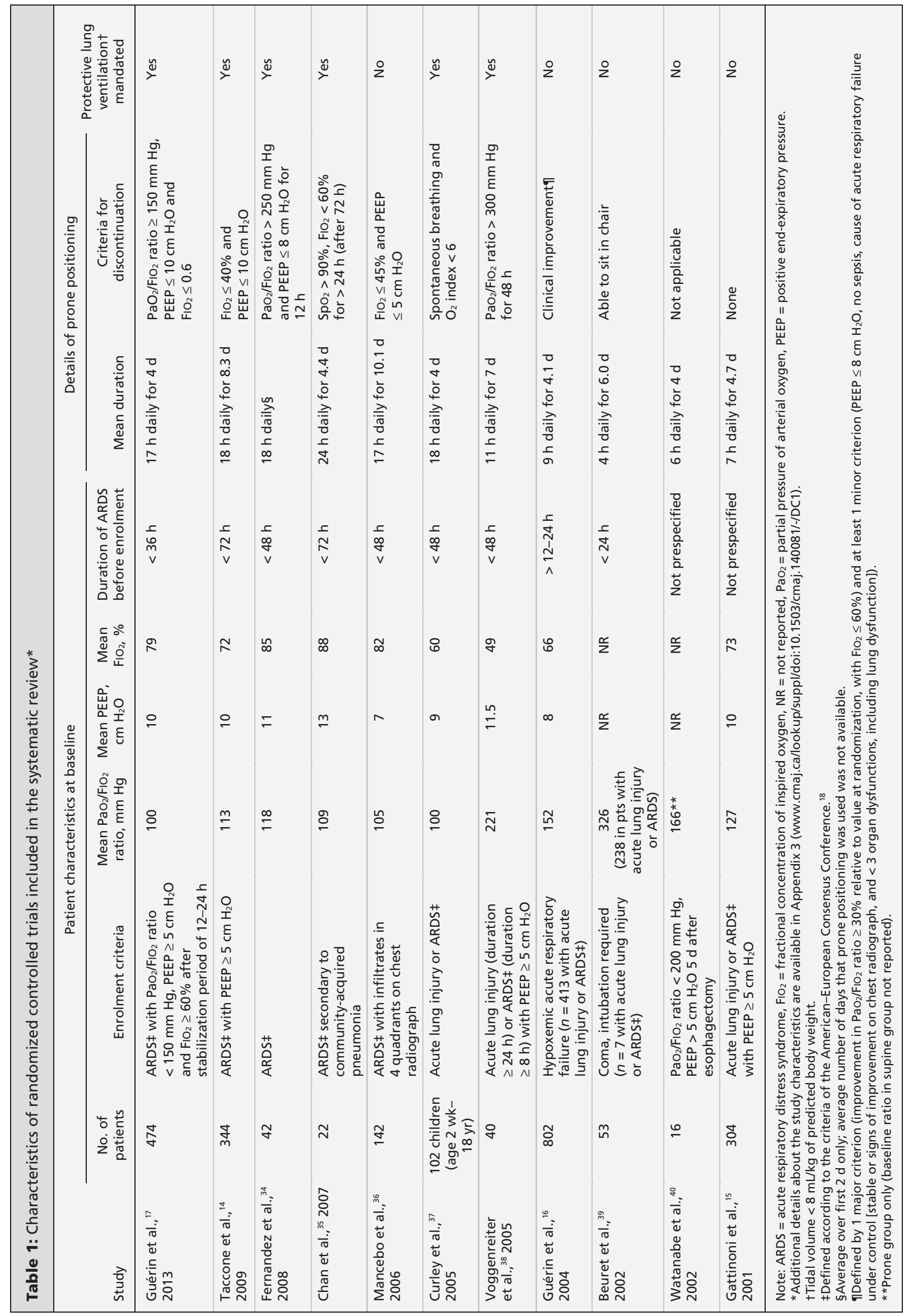


after a prespecified duration $\left(2\right.$ trials $\left.{ }^{15,40}\right)$. Six trials ( $n=1016)$ mandated protective lung ventilation in both study groups. Six studies ${ }^{14,16,17,34,36,39}$ permitted "rescue" prone positioning according to prespecified criteria for life-threatening hypoxemia in patients randomly assigned to the supine position. Protocols or guidelines for mechanical ventilation were used in 6 trials. ${ }^{14,17,35-38}$ Five trials used protocols to manage sedation ${ }^{17,34,36,37,40}$ and weaning from mechanical ventilation. ${ }^{16,17,34,36,37}$ Five of the 6 RCTs that mandated protective lung ventilation followed patients until discharge from hospital or a minimum of 90 days; the other trial ${ }^{37}$ followed patients until hospital discharge or 28 days, whichever occurred first.

The risk of bias was low in 6 trials, high in 2 and unclear in 3 (Table 2). Using GRADE methodol$\mathrm{ogy},{ }^{23}$ we found that the quality of evidence for the primary outcome of all-cause mortality was high (see Appendices 4 and 5, available at www.cmaj .ca/lookup/suppl/doi:10.1503/cmaj.140081/-/DC1). All of the RCTs analyzed outcomes by assigned group. Seven studies were ended early because of slow recruitment ${ }^{15,34-36,38,39}$ or on the basis of a stopping rule for futility. ${ }^{37}$ One trial at high risk of bias did not report mortality. ${ }^{40}$ For the primary outcome, there was no loss to follow-up in 4 trials..$^{17,35,37,38}$ In the remaining 6 trials, less than $5 \%$ of patients were lost to follow-up $\left(12 / 802,{ }^{16} 6 / 142,{ }^{36} 6 / 344,{ }^{14} 2 / 42,{ }^{34}\right.$ $2 / 53^{39}$ and $7 / 304^{15}$ ), and the proportion appeared balanced across treatment groups. Crossovers occurred in 8 trials $^{14-17,34,36,37,39}$ (range 4\%-32\% of all patients), which involved $0 \%-41 \%$ of patients initially assigned to prone positioning and 0\%-21\% of those assigned to the supine group.

There was no evidence of publication bias ( $p=0.4$ with Begg rank correlation test; $p=0.5$ with Peters regression test).

Table 2: Risk-of-bias assessment of the 11 trials included in the meta-analysis*

\begin{tabular}{|c|c|c|c|c|c|c|}
\hline Study & $\begin{array}{l}\text { Adequate } \\
\text { sequence } \\
\text { generation }\end{array}$ & $\begin{array}{l}\text { Concealment } \\
\text { of allocation }\end{array}$ & $\begin{array}{l}\text { Complete } \\
\text { outcome } \\
\text { reporting }\end{array}$ & $\begin{array}{c}\text { Complete } \\
\text { outcome data }\end{array}$ & $\begin{array}{l}\text { Trial ended } \\
\text { early (reason) }\end{array}$ & $\begin{array}{l}\text { Overall risk } \\
\text { of bias* }\end{array}$ \\
\hline Guérin et al., ${ }^{17} 2013$ & Yes & $\begin{array}{l}\text { Centralized, } \\
\text { Web-based }\end{array}$ & Yes & Yes & No & Low \\
\hline Taccone et al., ${ }^{14} 2009$ & Yes & Central & Yes & $\begin{array}{l}\text { No (mortality unknown } \\
\text { for } 1 / 175 \text { in supine group, } \\
1 / 169 \text { in prone group) }\end{array}$ & No & Low \\
\hline Fernandez et al., ${ }^{34} 2008$ & Yes & Central & Yes & $\begin{array}{l}\text { No (mortality unknown } \\
\text { for } 1 / 20 \text { in supine group, } \\
1 / 22 \text { in prone group) }\end{array}$ & $\begin{array}{l}\text { Yes (slow } \\
\text { enrolment) }\end{array}$ & Low \\
\hline Chan et al., ${ }^{35} 2007$ & Yes & $\begin{array}{l}\text { No (randomization } \\
\text { table visible to person } \\
\text { enrolling patients) }\end{array}$ & Yes & Yes & $\begin{array}{l}\text { Yes (slow } \\
\text { enrolment) }\end{array}$ & Hight \\
\hline Mancebo et al., ${ }^{36} 2006$ & Yes & $\begin{array}{l}\text { Sealed opaque } \\
\text { envelopes }\end{array}$ & Yes & $\begin{array}{l}\text { No (mortality unknown } \\
\text { for } 2 / 62 \text { in supine group, } \\
4 / 80 \text { in prone group) }\end{array}$ & $\begin{array}{l}\text { Yes (slow } \\
\text { enrolment) }\end{array}$ & Unclearł \\
\hline Curley et al., 372005 & Yes & $\begin{array}{l}\text { Sealed opaque } \\
\text { envelopes }\end{array}$ & Yes & Yes & $\begin{array}{l}\text { Yes (futility } \\
\text { stopping } \\
\text { rule) }\end{array}$ & Low \\
\hline $\begin{array}{l}\text { Voggenreiter et al., } \\
2005\end{array}$ & Yes & Central & Yes & Yes & $\begin{array}{l}\text { Yes (slow } \\
\text { enrolment) }\end{array}$ & Low \\
\hline Guérin et al., ${ }^{16} 2004$ & Yes & $\begin{array}{l}\text { Sealed opaque } \\
\text { envelopes }\end{array}$ & Yes & $\begin{array}{l}\text { No (mortality unknown } \\
\text { for } 7 / 385 \text { in supine group, } \\
4 / 417 \text { in prone group) }\end{array}$ & No & Unclearł \\
\hline Beuret et al.,, 2002 & Yes & $\begin{array}{l}\text { Sealed opaque } \\
\text { envelopes }\end{array}$ & Yes & $\begin{array}{l}\text { No (mortality unknown } \\
\text { for } 2 / 28 \text { in supine group, } \\
0 / 25 \text { in prone group) }\end{array}$ & $\begin{array}{l}\text { Yes (slow } \\
\text { enrolment) }\end{array}$ & Unclearł \\
\hline Watanabe et al., ${ }^{40} 2002$ & $\begin{array}{l}\text { No } \\
\text { (alternate } \\
\text { allocation) }\end{array}$ & $\begin{array}{l}\text { No } \\
\text { (alternate } \\
\text { allocation) }\end{array}$ & $\begin{array}{l}\text { No } \\
\text { mortality } \\
\text { data }\end{array}$ & NA & NR & Hight \\
\hline Gattinoni et al., ${ }^{15} 2001$ & Yes & Central & Yes & Yes & $\begin{array}{l}\text { Yes (slow } \\
\text { enrolment) }\end{array}$ & Low \\
\hline \multicolumn{7}{|c|}{ 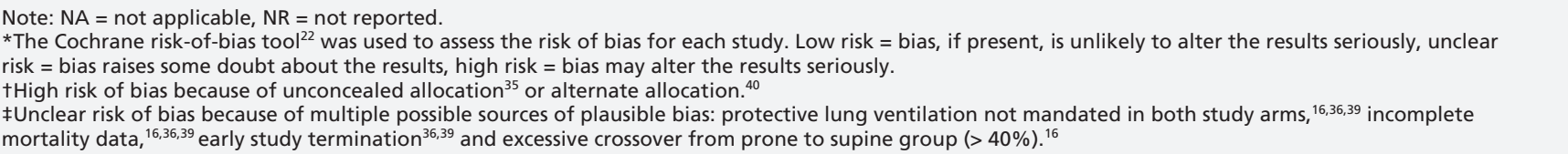 } \\
\hline
\end{tabular}




\section{Effect on mortality}

The 6 RCTs that mandated protective lung ventilation were included in the primary analysis. ${ }^{14,17,34,3,3,37,38}$ They all had a low risk of bias except one $\operatorname{trial}^{35}(n=22)$, which had a high risk of bias because allocation was not concealed. Mortality was reduced with the use of prone positioning (RR 0.74, 95\% CI 0.59-0.95; $I^{2}=29 \%$ ) compared with use of the supine position alone (Figure 2). Using a random-effects risk-difference model, we estimated that the number needed to treat to save 1 life was 11 (95\% CI 6-50). Our findings remained unchanged in several sensitivity analyses that tested alternative assumptions (Table 3). Conversely, there was no effect of prone positioning on mortality in the 4 trials that permitted higher tidal volumes than currently recommended (RR 0.98, 95\% CI 0.86-1.12; $I^{2}=0 \%$ ), which differed when compared with trials using protective lung ventilation (interaction $p=0.05$ ).

A priori subgroup analyses are summarized in Figure 3. All-cause mortality was reduced when the daily duration of prone positioning was prolonged (RR 0.77, 95\% CI $0.64-0.92 ; I^{2}=21 \%$ ) but not when the daily duration was shorter. Only 1 of the 6 trials with a prolonged duration did not use protective lung ventilation. ${ }^{36}$ Prone positioning reduced all-cause mortality among patients with severe hypoxemia at baseline (RR $0.76,95 \%$ CI $0.61-0.94 ; I^{2}=0 \%$ ). In the subgroups of patients with mild and moderate hypoxemia, prone positioning did not significantly reduce mortality, and statistical heterogeneity increased in the group with moderate hypoxemia $\left(I^{2}=42 \%\right)$. The test for interaction was significant for the analyses according to use of protective lung ventilation and daily duration of prone positioning, but not for the analysis according to degree of hypoxemia.

\section{Effect on secondary outcomes}

Improvements in oxygenation were greater in the prone group than in the supine group, with $\mathrm{PaO}_{2} / \mathrm{FIO}_{2}$ ratios increasing by $25 \%-36 \%$ during the first 3 days after randomization (Table 4). Moderate heterogeneity was detected for the analysis of $\mathrm{PaO}_{2} / \mathrm{FIO}_{2}$ ratio on day $1\left(I^{2}=49 \%\right)$ and day $2\left(I^{2}=27 \%\right)$, but not on day $3\left(I^{2}=0 \%\right)$.

The risk of pressure ulcers, obstruction of the endotracheal tube and dislodgement of the thoracostomy tube was higher among patients placed in the prone position than among those in the supine group. There was no difference in other adverse events between the 2 groups (Table 4 ).

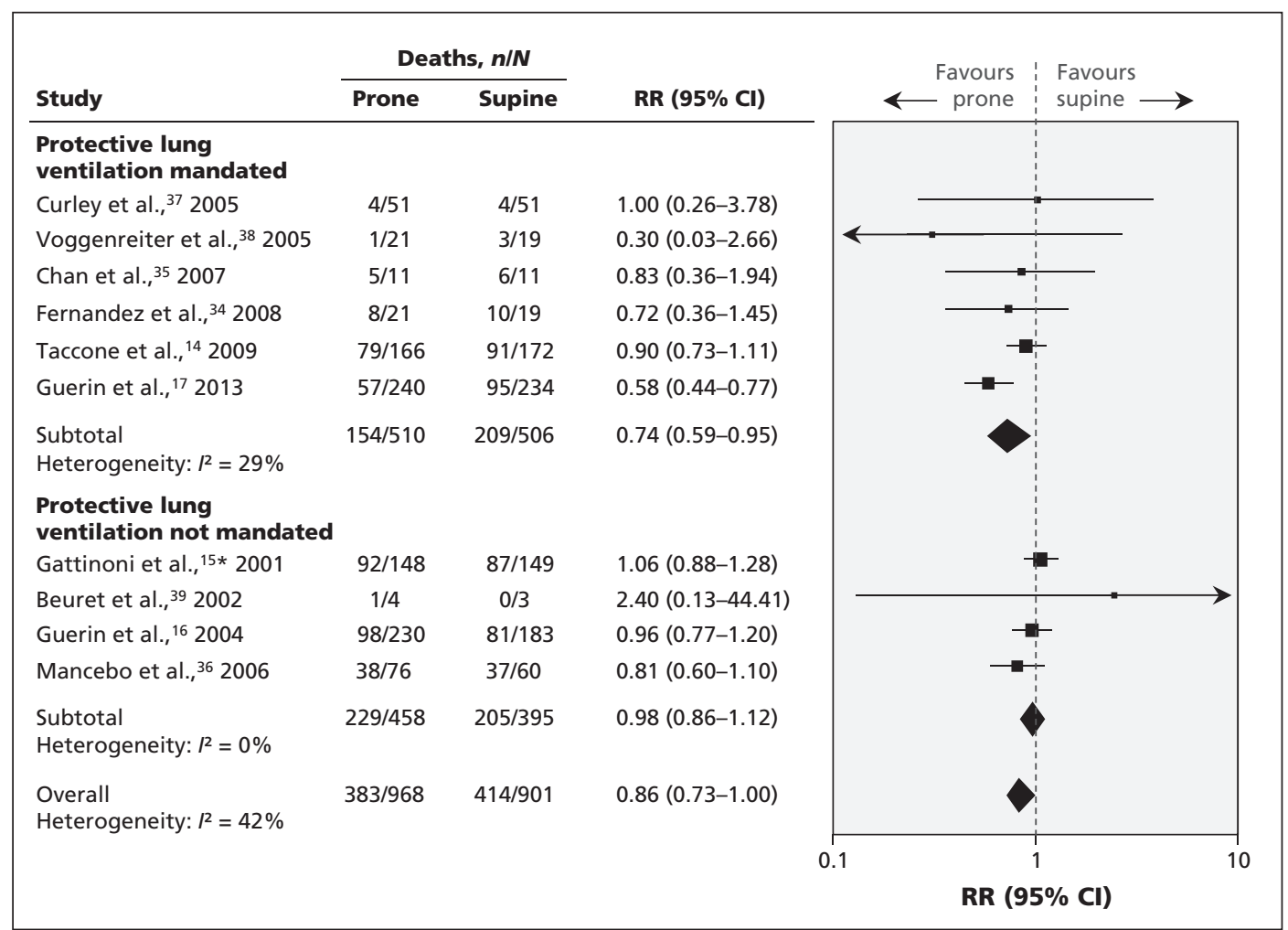

Figure 2: Effect of prone positioning during mechanical ventilation on all-cause mortality among patients with acute respiratory distress syndrome in trials that used protective lung ventilation (tidal volume $<8 \mathrm{~mL} / \mathrm{kg}$ ) and in trials that did not mandate protective ventilation. Risk ratios less than 1.0 indicate a decreased risk of death with prone positioning. * Mortality data differed from the original publication after verification by the primary investigator. The test for subgroup interaction is statistically significant $(p=0.05) . \mathrm{Cl}=$ confidence interval, $\mathrm{RR}=$ risk ratio. 


\section{Interpretation}

Our analysis of high-quality evidence showed that prone positioning during mechanical ventilation reduces mortality among patients with ARDS receiving protective lung ventilation. The quality of evidence was high, and the number needed to treat to save one life was $11(95 \% \mathrm{CI}$ 6-50). Our findings complement those of a recent positive $\mathrm{RCT}^{17}$ and showed consistency of effect across previous RCTs and in the sensitivity analyses.
Most RCTs of prone positioning during mechanical ventilation in patients with ARDS failed on their own to show statistically significant reductions in mortality despite improvements in oxygenation..$^{15,16,36,39}$ Previous systematic reviews were similarly unable to show reductions in mortality, ${ }^{41-43}$ although some suggested a mortality benefit among sicker patients. ${ }^{13,42}$ Limitations of earlier trials, including use of injurious tidal volumes ( $>8 \mathrm{~mL} / \mathrm{kg}$ of predicted body weight), enrolment of patients with mild ARDS, , 15,16,35,37-39 and inadequate duration of prone positioning, ${ }^{15,16,38,39}$

Table 3: Results of primary and sensitivity analyses for the effect of prone positioning during mechanical ventilation on mortality among patients with acute respiratory distress syndrome (ARDS)

\begin{tabular}{|c|c|c|c|c|}
\hline Analysis* & $\begin{array}{l}\text { No. of } \\
\text { trials }\end{array}$ & $\begin{array}{c}\text { No. of } \\
\text { deaths, } n / N\end{array}$ & $\begin{array}{l}\text { Risk ratio } \\
(95 \% \mathrm{Cl})\end{array}$ & $\begin{array}{l}I^{2} \text { value, } \\
\%\end{array}$ \\
\hline \multicolumn{5}{|l|}{ Primary } \\
\hline Trials mandating protective ventilation $\dagger$ & 6 & $363 / 1016$ & $0.74(0.59-0.95)$ & 29 \\
\hline \multicolumn{5}{|l|}{ Sensitivity } \\
\hline Included all trials $\ddagger$ & 10 & $797 / 1869$ & $0.86(0.73-1.00)$ & 42 \\
\hline Assumed patients lost to follow-up lived & 6 & $363 / 1020$ & $0.74(0.59-0.95)$ & 28 \\
\hline Assumed patients lost to follow-up died & 6 & $366 / 1020$ & $0.74(0.59-0.94)$ & 26 \\
\hline $\begin{array}{l}\text { Excluded trial in which allocation was not } \\
\text { concealed }^{35}\end{array}$ & 5 & $352 / 994$ & $0.73(0.55-0.98)$ & 43 \\
\hline Excluded trial with pediatric population ${ }^{37}$ & 5 & $355 / 914$ & $0.73(0.56-0.96)$ & 42 \\
\hline $\begin{array}{l}\text { Included trial that used moderate tidal } \\
\text { volume }(<10 \mathrm{~mL} / \mathrm{kg})^{36}\end{array}$ & 7 & $438 / 1152$ & $0.77(0.65-0.91)$ & 16 \\
\hline Fixed-effects model & 6 & $363 / 1016$ & $0.74(0.63-0.87)$ & 29 \\
\hline \multicolumn{5}{|c|}{$\begin{array}{l}\text { Note: } \mathrm{Cl}=\text { confidence interval. } \\
\text { * Random-effects models were used for all analyses except in the final sensitivity analysis. } \\
\text { †Tidal volume }<8 \mathrm{~mL} / \mathrm{kg} \text { of predicted or actual body weight. } \\
\text { fFor the } 2 \text { trials that enrolled some patients without ARDS, } 16,39 \text { we included only patients whose condition met the authors' } \\
\text { definition of ARDS; when the analysis was redone to include all patients in these trials, the risk ratio changed minimally }(0.87 \text {, } \\
\left.95 \% \mathrm{Cl} 0.74-1.02 ; I^{2}=48 \%\right)\end{array}$} \\
\hline
\end{tabular}

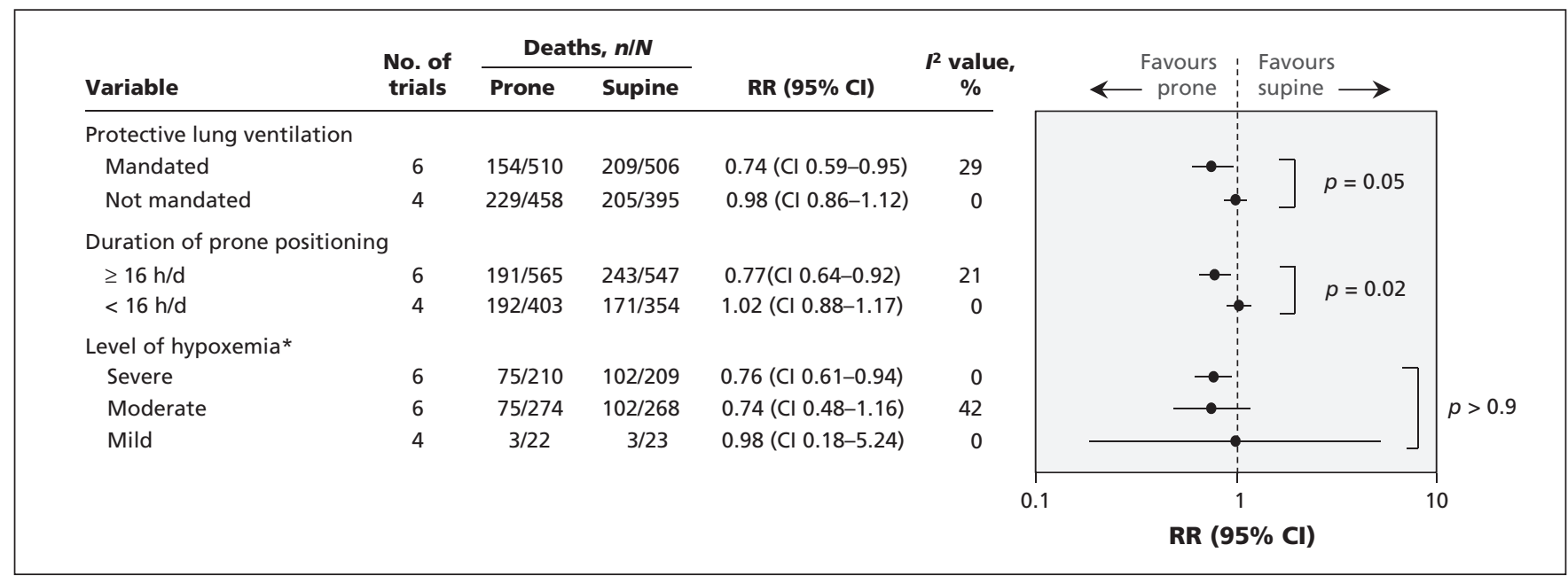

Figure 3: Effect of prone positioning during mechanical ventilation on all-cause mortality according to prespecified patient-level and trial-level subgroups. Risk ratios less than 1.0 indicate a decreased risk of death with prone positioning. *Severe hypoxemia = ratio of partial pressure of arterial oxygen to fraction of inspired oxygen $\left(\mathrm{PaO}_{2} / \mathrm{FlO}_{2}\right)<100 \mathrm{~mm} \mathrm{Hg}$; moderate $=\mathrm{PaO}_{2} / \mathrm{FlO}_{2}$ ratio $100-199 \mathrm{~mm} \mathrm{Hg}$; mild $=\mathrm{PaO}_{2} / \mathrm{FIO}_{2}$ ratio $200-299 \mathrm{~mm} \mathrm{Hg} . \mathrm{Cl}=95 \%$ confidence interval, RR = risk ratio. Baseline $\mathrm{PaO}_{2} / \mathrm{FlO}_{2}$ ratios were unavailable for 10 patients in 3 trials. ${ }^{17,34,35}$ 
may have reduced the "signal" and increased "noise." A recent RCT found a large reduction in mortality among patients with moderate to severe ARDS who were placed in the prone position for 16 hours per day and had protective lung ventilation. ${ }^{17}$ In our systematic review, we similarly reduced "noise" by limiting the primary analysis to trials mandating low tidal volumes and enrolling patients with moderate to severe ARDS; most trials also used long daily durations of prone positioning, which may have enhanced the "signal."

Our finding that prone positioning decreased mortality and improved oxygenation is consistent with results of prior observational and experimental studies, ${ }^{930,44}$ which showed that prone ventilation improves recruitment of collapsed alveoli. Use of the prone position reduced mortality in the subgroup of patients who had severe hypoxemia at baseline $\left(\mathrm{PaO}_{2} / \mathrm{FIO}_{2}\right.$ ratio $\left.<100 \mathrm{~mm} \mathrm{Hg}\right)$, with minimal statistical heterogeneity, a finding that is consistent with our previous systematic review. ${ }^{13}$ However, we found no evidence that the prone position had a differential effect according to severity of hypoxemia, acknowledging the limited number of patients with mild to moderate hypoxemia. Future trials may help to clarify the effects of prone positioning in patients with mild to moderate ARDS.
Prone positioning during mechanical ventilation is not without risks. Our study showed that patients in the prone group were at increased risk of pressure ulcers, obstruction of the endotracheal tube and dislodgement of the thoracostomy tube. Although there was no significant difference in the occurrence of other complications between the prone and supine groups, these adverse events may occur more frequently in centres with less experienced personnel who use prone positioning infrequently. Furthermore, the perceived risk of prone positioning and the impact on other aspects of critical care such as enteral feeding and sedation ${ }^{45-47}$ may prevent implementation of this manoeuvre in centres that do not frequently care for patients with severe ARDS. The increased risk of certain adverse outcomes underscores the need to have protocols for using prone positioning and to have adequate training and, when these are not available, to consider referring patients to centres with expertise. Future research is needed to address whether referring patients with severe ARDS early to experienced centres for prone positioning or other adjunctive therapies improves their outcomes. ${ }^{48,49}$

\section{Limitations}

Although we found high-quality evidence using rigorous methodology, our systematic review has limitations. Several trials were terminated early

Table 4: Physiologic, clinical and safety outcomes associated with prone positioning during mechanical ventilation

\begin{tabular}{|c|c|c|c|}
\hline Outcome & $\begin{array}{l}\text { No. of patients } \\
\text { or events }\end{array}$ & $\begin{array}{l}\text { Measure } \\
\text { of effect* }\end{array}$ & $\begin{array}{l}I^{2} \text { value, } \\
\%\end{array}$ \\
\hline Oxygenation $\left(\mathrm{PaO}_{2} / \mathrm{FlO}_{2}\right.$ ratio $) \dagger$ & No. of patients & Ratio of means $(95 \% \mathrm{Cl})$ & \\
\hline Day 1 & 1283 & $1.36(1.25-1.47)$ & 49 \\
\hline Day 2 & 1171 & $1.29(1.21-1.37)$ & 27 \\
\hline Day 3 & 933 & $1.25(1.18-1.31)$ & 0 \\
\hline Clinical and safety outcomes & No. of events, $n / N$ & Risk ratio $(95 \% \mathrm{Cl})$ & \\
\hline Ventilator-associated pneumonia & $368 / 1561$ & $0.89(0.71-1.13)$ & 0 \\
\hline Pressure ulcers & $818 / 1765$ & $1.27(1.16-1.40)$ & 0 \\
\hline Obstruction of endotracheal tube & $200 / 1847$ & $1.60(1.27-2.02)$ & 0 \\
\hline $\begin{array}{l}\text { Unplanned extubation or } \\
\text { dislodgement of endotracheal tube } \neq\end{array}$ & $211 / 2309$ & $1.08(0.78-1.48)$ & 16 \\
\hline $\begin{array}{l}\text { Unplanned removal of central or } \\
\text { arterial lines }\end{array}$ & $59 / 886$ & $1.49(0.42-5.27)$ & 67 \\
\hline Dislodgement of thoracostomy tube & $17 / 886$ & $3.14(1.02-9.69)$ & 0 \\
\hline Pneumothorax & $95 / 1663$ & $0.84(0.57-1.25)$ & 0 \\
\hline Cardiac arrest & $211 / 1527$ & $0.73(0.39-1.38)$ & 76 \\
\hline \multicolumn{4}{|c|}{$\begin{array}{l}\text { Note: } \mathrm{Cl}=\text { confidence interval, } \mathrm{PaO}_{2} / \mathrm{FIO}_{2} \text { ratio }=\text { ratio of partial pressure of arterial oxygen to fraction of inspired oxygen. } \\
\text { * Random-effects models were used for all analyses. } \\
\text { tWe measured effect on oxygenation by comparing the mean } \mathrm{PaO}_{2} / \mathrm{FIO}_{2} \text { ratio in the prone group to the closest available } \\
\text { recorded measurement in the supine group. If more than one measurement was taken, we chose the measurement closest to } \\
\text { the end of the session of prone positioning on that day. } \\
\text { fOne trial }{ }^{14} \text { included all dislodgements of endotracheal tubes, not just unplanned extubations. When we excluded the results of } \\
\text { this trial from the meta-analysis, the risk ratio for unplanned extubation was } 0.86\left(95 \% \mathrm{Cl} 0.62-1.20 ; l^{2}=0 \% ; 9 \text { trials, } 1471\right. \\
\text { patients, } 129 \text { events). }\end{array}$} \\
\hline
\end{tabular}


because of slow enrolment, which reduced statistical power. However, none of the trials was stopped early because of a beneficial effect. ${ }^{50}$

The trials were diverse with respect to inclusion criteria, daily duration of prone positioning and use of protocols for other aspects of ventilator management (e.g., weaning and sedation). Trials in which the daily duration of prone positioning was prolonged tended to be recent and overlapped with those that used protective lung ventilation. It was therefore difficult to identify the precise aspect of the patient population or the protocol for prone positioning most responsible for improved survival. Nevertheless, statistical heterogeneity was low for our primary outcome. Five of the 6 trials included in the primary analysis had risk ratios that pointed toward a benefit of prone positioning.

Several trials reported crossover of patients between the prone and supine groups. We analyzed all outcomes on an intention-to-treat basis, which would have underestimated the effect of prone positioning on mortality.

Although we searched for RCTs with either an adult or a pediatric study population, we found only one small trial that had enrolled children. An adequately powered RCT would be helpful to confirm our findings in children with ARDS.

Our findings are based on relatively few trials, some of which enrolled small numbers of patients and accrued few outcome events, which may have reduced precision and underestimated heterogeneity. The duration of follow-up in the included studies was short, and few examined the impact of prone positioning on long-term survival and quality of life.

Finally, all of the studies included in our primary analysis limited the duration of ARDS before enrolment, which made it difficult to ascertain whether prone positioning was beneficial if started late or as a rescue intervention for patients with life-threatening hypoxemia.

\section{Conclusion}

Our analysis of high-quality evidence showed that mechanical ventilation in the prone position significantly reduced mortality among patients with ARDS who received protective lung ventilation. This technique was beneficial to patients with moderate to severe ARDS when used for prolonged periods of 16 hours or more each day.

\section{References}

1. Ranieri VM, Suter PM, Tortorella C, et al. Effect of mechanical ventilation on inflammatory mediators in patients with acute respiratory distress syndrome: a randomized controlled trial. JAMA 1999;282:54-61.

2. Dreyfuss D, Soler P, Basset G, et al. High inflation pressure pulmonary edema. Respective effects of high airway pressure, high tidal volume, and positive end-expiratory pressure. Am Rev Respir Dis 1988;137:1159-64.
3. Muscedere JG, Mullen JB, Gan K, et al. Tidal ventilation at low airway pressures can augment lung injury. Am J Respir Crit Care Med 1994;149:1327-34.

4. Ventilation with lower tidal volumes as compared with traditional tidal volumes for acute lung injury and the acute respiratory distress syndrome. The Acute Respiratory Distress Syndrome Network. N Engl J Med 2000;342:1301-8.

5. Briel M, Meade M, Mercat A, et al. Higher vs lower positive end-expiratory pressure in patients with acute lung injury and acute respiratory distress syndrome: systematic review and meta-analysis. JAMA 2010;303:865-73.

6. Phua J, Badia JR, Adhikari NK, et al. Has mortality from acute respiratory distress syndrome decreased over time?: A systematic review. Am J Respir Crit Care Med 2009;179:220-7.

7. Stapleton RD, Wang BM, Hudson LD, et al. Causes and timing of death in patients with ARDS. Chest 2005;128:525-32.

8. Bryan AC. Conference on the scientific basis of respiratory therapy. Pulmonary physiotherapy in the pediatric age group. Comments of a devil's advocate. Am Rev Respir Dis 1974;110:143-4.

9. Galiatsou E, Kostanti E, Svarna E, et al. Prone position augments recruitment and prevents alveolar overinflation in acute lung injury. Am J Respir Crit Care Med 2006;174:187-97.

10. Gattinoni L, Protti A. Ventilation in the prone position: For some but not for all? CMAJ 2008;178:1174-6.

11. Albert RK, Hubmayr RD. The prone position eliminates compression of the lungs by the heart. Am J Respir Crit Care Med 2000;161:1660-5.

12. Lamm WJ, Graham MM, Albert RK. Mechanism by which the prone position improves oxygenation in acute lung injury. Am J Respir Crit Care Med 1994;150:184-93.

13. Sud S, Friedrich JO, Taccone P, et al. Prone ventilation reduces mortality in patients with acute respiratory failure and severe hypoxemia: systematic review and meta-analysis. Intensive Care Med 2010;36:585-99.

14. Taccone P, Pesenti A, Latini R, et al. Prone positioning in patients with moderate and severe acute respiratory distress syndrome: a randomized controlled trial. JAMA 2009;302:1977-84.

15. Gattinoni L, Tognoni G, Pesenti A, et al. Effect of prone positioning on the survival of patients with acute respiratory failure. N Engl J Med 2001;345:568-73.

16. Guerin C, Gaillard S, Lemasson S, et al. Effects of systematic prone positioning in hypoxemic acute respiratory failure: a randomized controlled trial. JAMA 2004;292:2379-87.

17. Guérin C, Reignier J, Richard JC, et al. Prone positioning in severe acute respiratory distress syndrome. N Engl J Med 2013; 368:2159-68.

18. Bernard GR, Artigas A, Brigham KL, et al. The AmericanEuropean Consensus Conference on ARDS. Definitions, mechanisms, relevant outcomes, and clinical trial coordination. Am J Respir Crit Care Med 1994;149:818-24.

19. Ranieri VM, Rubenfeld GD, Thompson BT, et al. Acute respiratory distress syndrome: the Berlin definition. JAMA 2012;307: 2526-33.

20. Sud S, Sud M, Friedrich JO, et al. High-frequency ventilation versus conventional ventilation for treatment of acute lung injury and acute respiratory distress syndrome. Cochrane Database Syst Rev 2013;CD004085.

21. Sud S, Sud M, Friedrich JO, et al. High frequency oscillation in patients with acute lung injury and acute respiratory distress syndrome (ARDS): systematic review and meta-analysis. BMJ 2010;340:c2327.

22. Higgins JP, Altman DG, Gøtzsche PC, et al. The Cochrane Collaboration's tool for assessing risk of bias in randomised trials. BMJ 2011;343:d5928.

23. Schünemann HJ, Oxman AD, Brozek J, et al. Grading quality of evidence and strength of recommendations for diagnostic tests and strategies. BMJ 2008;336:1106-10.

24. Friedrich JO, Adhikari NK, Beyene J. The ratio of means method as an alternative to mean differences for analyzing continuous outcome variables in meta-analysis: a simulation study. BMC Med Res Methodol 2008;8:32.

25. Higgins JP, Thompson SG. Quantifying heterogeneity in a metaanalysis. Stat Med 2002;21:1539-58.

26. Begg CB, Mazumdar M. Operating characteristics of a rank correlation test for publication bias. Biometrics 1994;50:1088-101.

27. Peters JL, Sutton AJ, Jones DR, et al. Comparison of two methods to detect publication bias in meta-analysis. JAMA 2006;295: 676-80.

28. Leal RP, Gonzalez R, Gaona C, et al. Randomized trial compare prone vs supine position in patients with ARDS [abstract]. Am J Respir Crit Care Med 1997;155:A745.

29. Gaillard S, Guerin C, Avzac L, et al. A multicenter randomized controlled trial of prone position in acute respiratory failure [abstract]. Am J Respir Crit Care Med 2000;161:A380. 
30. Papazian L, Gainnier M, Marin V, et al. Comparison of prone positioning and high-frequency oscillatory ventilation in patients with acute respiratory distress syndrome. Crit Care Med 2005;33: 2162-71.

31. Demory D, Michelet P, Arnal JM, et al. High-frequency oscillatory ventilation following prone positioning prevents a further impairment in oxygenation. Crit Care Med 2007;35:106-11.

32. Ibrahim TS, El-Mohamady HS. Inhaled nitric oxide and prone position: How far they can improve oxygenation in pediatric patients with acute respiratory distress syndrome? J Med Sci 2007;7:390-5.

33. Wang WX, Xu B, Ma HS, et al. Effect of prone position on the oxygenation of patients with acute respiratory distress syndrome after failure recruitment maneuvers at high altitudes [article in Chinese]. Zhongguo Wei Zhong Bing Ji Jiu Yi Xue 2012;24:596-9.

34. Fernandez R, Trenchs X, Klamburg J, et al. Prone positioning in acute respiratory distress syndrome: a multicenter randomized clinical trial. Intensive Care Med 2008;34:1487-91.

35. Chan MC, Hsu JY, Liu HH, et al. Effects of prone position on inflammatory markers in patients with ARDS due to communityacquired pneumonia. J Formos Med Assoc 2007;106:708-16.

36. Mancebo J, Fernandez R, Blanch L, et al. A multicenter trial of prolonged prone ventilation in severe acute respiratory distress syndrome. Am J Respir Crit Care Med 2006;173:1233-9.

37. Curley MA, Hibberd PL, Fineman LD, et al. Effect of prone positioning on clinical outcomes in children with acute lung injury: a randomized controlled trial. JAMA 2005;294:229-37.

38. Voggenreiter G, Aufmkolk M, Stiletto RJ, et al. Prone positioning improves oxygenation in post-traumatic lung injury - a prospective randomized trial. J Trauma 2005;59:333-41, discussion 341-3.

39. Beuret P, Carton MJ, Nourdine K, et al. Prone position as prevention of lung injury in comatose patients: a prospective, randomized, controlled study. Intensive Care Med 2002;28:564-9.

40. Watanabe I, Fujihara H, Sato K, et al. Beneficial effect of a prone position for patients with hypoxemia after transthoracic esophagectomy. Crit Care Med 2002;30:1799-802.

41. Abroug F, Ouanes-Besbes L, Elatrous S, et al. The effect of prone positioning in acute respiratory distress syndrome or acute lung injury: a meta-analysis. Areas of uncertainty and recommendations for research. Intensive Care Med 2008;34:1002-11.

42. Alsaghir AH, Martin CM. Effect of prone positioning in patients with acute respiratory distress syndrome: a meta-analysis. Crit Care Med 2008;36:603-9.

43. Sud S, Sud M, Friedrich JO, et al. Effect of mechanical ventilation in the prone position on clinical outcomes in patients with acute hypoxemic respiratory failure: a systematic review and meta-analysis. CMAJ 2008;178:1153-61

44. Gattinoni L, Pesenti A. The concept of "baby lung." Intensive Care Med 2005;31:776-84.

45. Léonet S, Fontaine C, Moraine JJ, et al. Prone positioning in acute respiratory failure: survey of Belgian ICU nurses. Intensive Care Med 2002;28:576-80.

46. Bein T, Ritzka M, Schmidt F, et al. Positioning therapy in intensive care medicine in Germany. Results of a national survey [article in German]. Anaesthesist 2007;56:226-31.

47. Hudack ME. Prone positioning for patients with ARDS. Nurse Pract 2013;38:10-2.

48. Kahn JM, Goss CH, Heagerty PJ, et al. Hospital volume and the outcomes of mechanical ventilation. N Engl J Med 2006;355:41-50.

49. Peek GJ, Mugford M, Tiruvoipati R, et al. Efficacy and economic assessment of conventional ventilatory support versus extracorporeal membrane oxygenation for severe adult respiratory failure (CESAR): a multicentre randomised controlled trial. Lancet 2009;374:1351-63.

50. Montori VM, Devereaux PJ, Adhikari NK, et al. Randomized trials stopped early for benefit: a systematic review. JAMA 2005; 294:2203-9.
Affiliations: Institute for Better Health and Division of Critical Care (S. Sud), Department of Medicine, Trillium Health Partners, University of Toronto, Mississauga, Ont.; Interdepartmental Division of Critical Care (S. Sud, Friedrich, Adhikari), University of Toronto, Toronto, Ont.; Departments of Critical Care and Medicine, and Li Ka Shing Knowledge Institute (Friedrich), St. Michael's Hospital, Toronto, Ont.; Dipartimento di Anestesia e Rianimazione (Taccone, Gattinoni, Polli), Fondazione IRCCS - Ospedale Maggiore Policlinico, Mangiagalli e Regina Elena, Milan, Italy; Istituto di Anestesiologia e Rianimazione (Polli, Gattinoni), Università degli Studi di Milano, Milan, Italy; Dipartimento di Fisiopatologia Medico-Chirurgica e dei Trapianti (Gattinoni), Fondazione IRCCS Ca' Granda - Ospedale Maggiore Policlinico, Università degli Studi di Milano, Milan, Italy; Department of Critical Care Medicine (Adhikari), Sunnybrook Health Sciences Centre, Sunnybrook Research Institute, Toronto, Ont.; Department of Cardiovascular Research (Latini), Istituto di Ricerche Farmacologiche Mario Negri, Milan, Italy; Dipartimento di Medicina Perioperatoria e Terapie Intensive (Pesenti), Azienda Ospedaliera San Gerardo, Monza, Italy; Dipartimento di Medicina Sperimentale (Pesenti), Università degli Studi di Milano-Bicocca, Milan, Italy; Service de Réanimation Médicale et Assistance Respiratoire (Guérin), Hôpital de la Croix-Rousse, Lyon, France; Servei de Medicina Intensiva (Mancebo), Hospital de Sant Pau, Barcelona, Spain; University of Pennsylvania School of Nursing (Curley), Philadelphia, Pa.; Intensive Care Unit (Fernandez), Hospital Sant Joan de Deu - Fundacio Althaia, CIBERES, Manresa, Spain; Universitat Internacional de Catalunya (Fernandez), Barcelona, Spain; Section of Chest Medicine (Chan), Department of Internal Medicine, Taichung Veterans General Hospital, Taichung, Taiwan; Institute of Physiology (Chan), National Yang-Ming University, Taipei, Taiwan; Service de Réanimation (Beuret), Centre Hospitalier, Roanne, France; Department of Orthopaedic and Trauma Surgery (Voggenreiter), Hospitals in the Natureparc Altmühltal, Eichstätt, Germany; Faculty of Medicine (M. Sud), University of Toronto, Toronto, Ont.; Consorzio Mario Negri Sud (Tognoni), Santa Maria Imbaro, Italy.

Contributors: Sachin Sud had full access to all of the data and takes responsibility for the integrity of the data and the accuracy of the analysis. He contributed to the study concept and design and to the acquisition, analysis and interpretation of data. Claude Guerin, Jan Friedrich and Neill Adhikari contributed to the study concept and design. Jan Friedrich, Neill Adhikari and Maneesh Sud contributed to the acquisition, analysis and interpretation of data. Sachin Sud drafted the manuscript. All of the authors critically revised the manuscript for important intellectual content and approved the final version submitted for publication. All of the authors agreed to act as guarantors of the work.

Funding: No specific funding was received for this study. Jan Friedrich is a clinician-scientist of the Canadian Institutes of Health Research (CIHR). Martha Curley's work was funded by the National Institutes of Health/National Institute of Nursing Research (NIH/NINR) (grant no. RO1NR05336). 